\title{
Sustainable Development and Diversifying Competencies Curricula
}

This paper explores the question how to approach thinking about curriculum construction for European engineering schools in the age of sustainable development. We present a theoretical argument that curriculum thinkers need to broaden their focus from the "restricted competences" paradigm (RCP) in curriculum thinking to consider how to make curricula within a diversifying competences paradigm (DCP). We claim that the best response to the challenge of sustainability is to produce more skill-diversity among engineers while simultaneously training engineers to make the most of this diversity. We support this claim with two arguments. First, we explore the problem-solving power of diversely skilled collectives, suggesting that this increases relative to homogenous collectives when confronting complex problems. Then we show that sustainable development is not only a complex problem, but an extremely complex or wicked problem. Based on these two conclusions, we propose a mixed-medium curricular model which illustrates how engineering schools might be reformed in order to produce greater student competence diversification.

Keywords: sustainability education, complexity, diversity, curriculum theory, media studies approaches to curricula, competence-based education

\section{Acknowledgement}

The authors would like to acknowledge the support of the EU Erasmus+ funding body under grant number 2018-1-FR01-KA203-047854 and to thank both our colleagues at the ENSTA Bretagne and the members of project A-STEP 2030 for their support, feedback, and intellectual stimulation.

The European Commission support for the production of this publication does not constitute an endorsement of the contents, which reflect the views only of the authors, and the Commission cannot be held responsible for any use which may be made of the information contained therein.

\section{Author Information}

Brad Tabas, ENSTA Bretagne, France; https://orcid.org/0000-0003-0156-8389

Klara Kövesi, ENSTA Bretagne, France; https://orcid.org/0000-0002-4036-6475

\section{How to cite this article:}

Tabas, Brad, Klara Kövesi. "Sustainable Development and Diversifying Competencies Curricula.” Információs Társadalom XX, no. 2 (2020): 118-131. 


\section{Introduction}

Sustainable development is a keyword in contemporary European discussions on the future of engineering education. This paper aims to contribute to that discussion by exploring the idea that increasing competence diversity among engineers is a means of increasing their collective problem-solving capacity, which would in turn assist in the struggle to develop sustainably. This paper begins by expounding the argument that greater diversity amounts to greater problem-solving potential, simultaneously indicating ways in which the implications of this argument challenge hegemonic thinking on sustainability education for engineers. In the second half of the paper, we explore in more concrete terms what taking diversity seriously might mean for the future of engineering education, presenting a mixed media model curriculum is aimed at improving skills diversification relative to current norms. Our overall aim is not to present a study of what exists but to propose a possible model for future curricula that might inform future sustainability-oriented innovations in European engineering curricula.

\section{Restricted competences}

Curriculum theorists typically consider the minimum set of subjects or competences that each and every student ought to acquire during their studies. We call this the restricted competences paradigm (RCP) of curriculum thinking. One of the recent trends in RCP thinking is an attempt to revise core curricula to include sustainable development and RRI competences. In the case of the CTI, the body overseeing the accreditation of French and many other francophone countries' engineering degrees, these include at least three core competences: a) the capacity to identify ethical and professional responsibilities; b) the capacity to take into account environmental challenges; c) the capacity to take into account social impacts. A recent literature survey (Tabas et al. 2019) carried out in the context of the European project A-STEP 2030 (Attracting Diverse Talent to the Engineering Profession) reveals that almost all of the research on training engineers for sustainable engineering similarly operate within an RCP framework. There are many reasons why such a restricted approach is attractive, including clarity, simplicity, and practicality. Other reasons for pursuing this approach include institutional legitimization and a desire for standardization, often tied to the perceived need to meet or establish national and international certification standards (for instance those of the CTI, ENAEE, EI, etc.), or a need to justify point by point the contents of curricula to companies, students, and other social actors. Yet it must also be said that the RCP approach sets out to establish a minimum action towards incorporating sustainability within their curricula. It must also be noted that RCP approaches implicitly assume that engineers will be confronting sustainable 
development challenges alone, to the extent that each and every student is prepared with the skills required for individually confronting development. ${ }^{1}$ Yet it remains worthwhile to pose the question of whether meeting the minimum requirements for addressing sustainability is the same thing as doing what is best for preparing future members of the engineering profession to address sustainability. We suggest that thinking about sustainable development curricula within what we call the diversifying competences paradigm (DCP) may help future curricula come closer to this optimum.

\section{Diversifying Competences}

A DCP curriculum does not aim to train students in a standard minimum number of required competences, rather it aims to maximize the total diversification of the portfolio of competences acquired by the student body as a whole. By competences, we intend skills of all sorts, both math and science skills as well as skills such as language and leadership skills. By diversity and competence diversity we do not primarily have in mind ethnic or gender diversity, even if these kinds of input diversities matter for producing curricula with significant output diversity. Competence diversity as we understand it involves the possession of different sets of competences or differing cognitive portfolios: different viewpoints and ways of describing and modelling problems, different bodies of knowledge, different heuristics and problem-applicable tools, etc.. Diversity is not randomness. For competence differences to matter for engineers, they must be relevant to sustainability problems: differences that make a difference.

Why foster competence diversity?

Diversity trumps homogeneity when confronting complex problems. This has been demonstrated across numerous domains ranging from empirical studies to computer models. This point has been made in general terms by Surowiecki (2005) and Scott Page (2007, 2010, and 2019). In ecology, diversity has been linked to the robustness of ecological systems (Krakauer 2006; Olivier et al. 2015; Whitacre and Bender 2010). The business case for diversity has been extensively documented (Robinson and Dechant 1997; Richard 2000; Erhardt et al. 2003). Diversity also positively correlates with increases in collective creativity and innovation capacity (Sawyer 2007; McLeod et al. 1996; Livermore 2016).

Why does diversity work? One answer has to do with cognitive capacity: each individual can only master a certain range of things. Yet when cognitive

\footnotetext{
1 This is because different problem-solving logics apply to individuals and groups. One recent study, Quelhas, et al. (2019) explicitly states that they conceive of engineering problem solving as an individual activity. As they put it, they seek to isolate: "the competencies that can contribute to the formation of an engineer capable of bringing adequate solutions to the conflicts of sustainability in the twenty-first century." Note the usage the singular here.
} 
power is pooled, it can be greater than that possessed by any individual. But this does not directly explain why diverse groups beat homogenous ones. Page (2010) offers two explanations for the power of diversity.

The first explanation offered by Page involves what he calls "averaging". He shows that it is on average statistically more probable that one of the tools will be adapted to any given situation when you have lots of types of problem-solving expertise (i.e. lots of diversity within your group) than if you have less diversity. The second explanation that he offers he calls "diminishing returns to type". The diminishing returns hypothesis argues that like-minded problem solvers each have relatively less to add to solving a problem than does a diverse group, because the additive synergy of like-minded problem solvers' capacities is less than that of a diverse group.

In reality, if we can predict which problems we will encounter, and we can specify clearly what will count as adequate solutions, then we have little reason to cultivate diversity. Diverse groups only manifest their superior problem-solving abilities when confronting unexpected or highly complex problems. For example, the power of "averaging" is manifest when unpredictable systems serve up simple problems: a broader set of tools is most likely to possess one that fits each situation. The "diminishing returns" argument is relevant when confronting highly complex problems which demand creative and innovative solutions (innovation is here understood as the power of finding relevant and novel connections between elements (Runco and Jaeger 2012).

What kind of problem is sustainable development? As we will argue in the following, it is a problem of the highest thinkable degree of complexity, hence one where competence diversity among problem solvers is desirable.

\section{Comparing RCP to DCP}

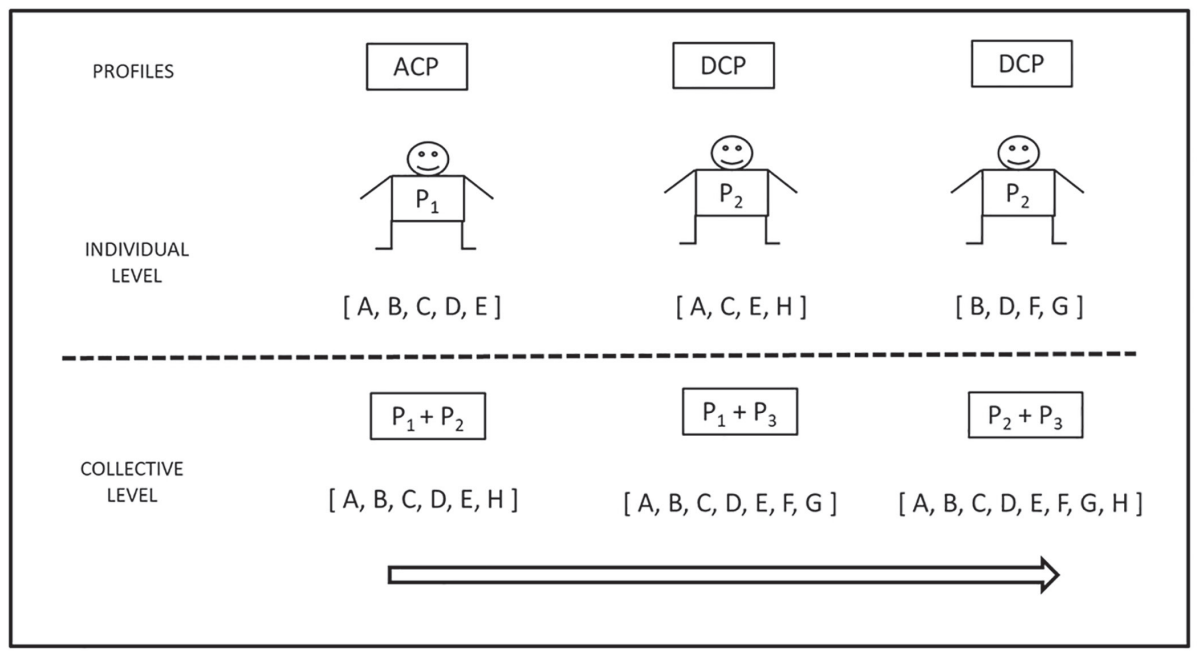

Figure 1. Competence diversity model 
Before exploring the hyper-complexity of sustainability, let us recapitulate by illustrating the differences between ideal student outputs of restricted and diversifying competence logics.

The three engineers have different "capacity" profiles. P1 has a broad and highly rationalized set of skills-they correspond to the key skills isolated by RCP theories. P2 and P3 have differing and superficially less "organized" sets of skills corresponding to theoretical DCP outputs (we illustrate P2 and P3 as having fewer individual skills than P1 for demonstration's sake. In reality, P1, P2, P3 could possess quantitatively equal capacity portfolios. In reality, too, the connections between the DCP skill sets are just as "logical" as that of the A-B-C organization of the RCP skills-their connective logic is simply less evident). What the model shows is that when collaborating, P2 and P3 combine to bring more knowledge and problem-solving capacities than P1 or than any collective of P1s.

\section{Sustainable Development as a Wicked Problem}

The value the DCP paradigm depends upon whether sustainable development is a complex problem that will throw up unanticipated challenges and which will call for significant creativity and innovation. Echoing a number of commentators, we submit that attempting to engineer sustainable development involves tangling with a supremely complex problem, even a "wicked problem" (WP).

WPs are the very apotheosis of complex problems. This is a simplified statement of Rittel and Webber's initial definition (1973). For them, WPs have 10 key characteristics: 1) No definitive formulation; 2) No 'stopping rule'; 3) No 'true-false' solutions; 4) no 'ultimate' tests of a solutions efficacity; 5) every attempted solution also creates problems; 6) WPs cannot be exhaustively described nor resolved; 7) are singular; 8) are part of an entangled web of problems; 9) any proposed solutions only fix part of the problem; 10) erroneous solutions are consequential.

Sustainable development is a WP. Suppose we try to formulate the problem of sustainable development as an equation, as is done in Elkington's “triple bottom line” approach to sustainability (Elkington and Rowlands 1999). A "smart" technical like Industry 4.0 may initially seem to address sustainability perfectly through a neat balancing of the triple bottom line. Industry 4.0 can increase efficiency, lower costs and reduce waste. According to Elkington's formula, this balances economics and environment. Industry 4.0 would lower production costs, giving customers access to better products, a social positive. Industry 4.0 seems like a win-win-win proposition from the point of view sustainability. Yet looking again within a longer time frame will reveal the wickedness of the problem (aspect 9). While smart factories may lower the relative environmental burden by reducing waste, they also encourage and reinforce path dependence (aspect 5). The production efficiencies that they promise may reduce the waste consumption of certain key resources, but economic histori- 
ans have noted that this will likely lead to a global expansion of resource consumption, as reduced demand leads to lower resource prices, which in turn prompts innovators to seek new forms of resource exploitation (aspect 8). One might also consider environmental externalities (aspect 2) such as Industry 4.0's reliance on coltan, a currently unsustainable resource (Nest 2011). From a social point of view, Industry 4.0 is likely to create job losses, and it is possible that it could likewise contribute to undermining our environmental future (aspect 10). All of these complexifications reveal the wickedness of sustainable development as a problem, and the danger in accepting any simple formulation of sustainability problems (aspect 1). Sustainable development problems (not to mention sustainable development as a problem) are maximally complex and escape from neat true-false solutions (aspect 3 ).

That said, WPs can be dealt with in better and worse ways, and approaching WPs with a diverse collective of problem solvers is one way of increasing the probability of having better rather than worse outcomes.

\section{Challenges Associated with Creating Diversifying Curricula}

If it is desirable to generate diverse collectives of engineers, making up a curriculum that is capable of fostering diversity is far from obvious. A diversifying curriculum is not a chaotic mangle. Students acquire different skills and cultivate different perspectives, but these must track with real world needs. A diversifying curriculum must be able to generate differences that will make a difference for confronting real-world problems, and it must be able to train differently skilled actors that are capable of working together.

Any attempt to generate curricular diversity will have to meet the following criteria:

\subsection{Feasibility}

The feasibility of a diversifying curriculum is far from obvious within a traditional institution. Most engineering schools host a limited number of classrooms and teachers, and they generally stipulate a minimum number of students for each course. Decreasing class sizes and teacher numbers increases student competence diversity, but it also raises costs and increases scheduling difficulties. In short, the limitations on the instructional medium of the brick and mortal institution imply that almost all students following any specialization at the school will come out having very similar skill sets: most will have learned the same skills in the same lectures taught by the same teachers. Increasing the differentiation of student outputs can only be accomplished with significant cost inputs.

Another related feasibility concern involves accreditation and examination. Non-standard outcomes challenge the expected comparability of degrees. One of 
the central feasibility challenges for any diversifying curriculum is to build in quality control, oversight, and comparability mechanisms which permit employers and others to identify student success and engagement in a meaningful way.

\subsection{Optimizing Collaboration among Different Actors}

One of the advantages of an RCP format is that it has a clear core that assures that each individual can work independently. DCP schools also need a core curriculum, but this must be aimed towards teaching students how to learn how to learn independently as well as training them in the skills required to work collectively. Some key skills necessary for optimizing collective productivity might include:

A. Common Orientation: The word curriculum comes from the Latin word for path, and RCP curricula provide students with a strong narrative orientation because every element in the curriculum has been selected by administrators as part of a single developmental narrative. DCP curricula challenge or obscure this institutional meta-narrative, by allowing each student to follow and independent competence development logic. One of the requirements for effective collaboration among diverse actors is the possession of common objectives and values. Fostering collective narrative orientation within difference and complexity is possible, but diversifying curricula need to make a particular effort to encourage students to generate meta-narratives which reinforce intersections between their individual and collective teleologies. One keystone of this orientation would be the cultivation of sustainable values. Despite the hopes that may be placed in green markets, it is important to recognize that sustainability will only be achieved if we value it. For this reason alone, students need to be encouraged to care about sustainable development as a project.

B. Collaboration Skills: Profiting from diversity requires that core curricula teach diverse individuals how to work together. Many engineering schools see diversity as a problem, not a solution. In part this explains the preference in RCP theorizing for imagining engineers as autonomous actors. Diverse groups can only take advantage their diversity if they are inclusive. According to Mor Barak (2015), a group is inclusive if all individuals feel that they are part of both formal and informal collective activities. This cannot happen if team members suffer from the epistemic or hermeneutic injustice described by Fricker (2007). It will not happen if diverse groups submit to group think (Surowiecki 2005; Sunstein and Hastie 2015). Avoiding these traps requires specifically training students to become aware of the cognitive biases and social habits that currently reinforce non-inclusion and poor collaboration.

C. Seminal Skills Training: Students in DCP programs need to be capable of learning for themselves. Yet even if each student will head towards a different endpoint within the curriculum, identifying and training students in certain basic skills remains necessary. Nelson and Kosselyn (2017) have identified that some skills are seminal, meaning that they are like seeds that later will permit 
self-directed learning and competence acquisition. One can illustrate this notion of seminal skill through the example of alphabetization. Learning to read allows one to learn many other things through reading, though the right of passage into this wide domain of self-empowered learning is the ability to recognize letters, their sounds, and how they fit together to form words. DCP curricula need to introduce students to seminal skills that will best begin learning for themselves via experimentation within the general framework of their specialization.

\section{A DCP Model Curriculum}

To illustrate how a DCP curriculum might function in practice, we now present a model DCP curriculum. Our presentation of this curriculum is influenced by media studies, and in particular by the idea that different educational media are best for encouraging different types of competence development. Our model illustrates how we can go beyond the traditional curricular medium of the brick and mortar school to make new levels of student competence diversification possible. Though this model is theoretical, we have been inspired by the curriculum of Charles Sturt University Engineering (a school cited by a recent MIT global study as one of the world leaders in engineering education (Graham 2018) - we mention this to encourage interested readers to perform empirical case studies.)

\subsection{Overview}

Our DCP curricular model is based around the complimentary integration of three medially differentiated approaches to education: 1) Project-Based Learning (PBL); 2) Internet-Based Learning (IBL); 3) Classroom Learning (CL).

\subsubsection{PBL}

PBL is a good medium for fostering diverse competences. Through in-school challenges, in-company internships and off-site humanitarian interventions, PBL allows each student to develop a unique skill set based on their individual experiences, also allowing each student to validate the value of difference by confronting the same challenges in different ways. Engaging in team challenges can help students to develop core teamwork competences by playing different but complimentary roles within their teams. PBL ensures not only talent diversification but also skill relevance.

\subsubsection{IBL}

In complement with PBL, our model replaces lecture-based courses with IBL. While completing their internships, students can remotely follow lectures and 
validate their mastery the lessons learned using online testing tools. The IBL component can offer a vast range of online tutorials. At CSU, for example, students are required to master 200 core competences and 400 elective competence modules (600 in total) out of more than 1000 options. Given the massive stocking abilities of servers, the total number of competence modules available would expand, particularly if resources were shared across institutions. IBL appropriate subjects are those that can be explained and evaluated according to simple problem-solving heuristics. By simple we do not mean basic or introductory, but rather such skills whereby true-false evaluation is possible (this is rarely the case with values-based, interpersonal, and reflective skills, for example). IBL appropriate subjects mostly overlap with subjects currently taught as lecture courses with multiple-choice or similar evaluations. Replacing traditional lectures with IBL will not necessarily impact educational quality. As Clayton Christensen (2010) has observed, one of the advantages of the new kinds of scaling made possible by IBL learning is that classes can be tailored to specific kinds of learners (visual, auditory, kinesthetic...). As a medium, IBL permits delocalization and temporal openness. This means that students can choose their IBL modules based upon challenges encountered in their PBL curriculum, and once having completed their IBL exams they can employ the competences that they have acquired in concrete situations, which will allow them to master and to reinforce what they have learned. Morgan and Lindsay (2016) call this a "just-in-time” approach to learning rather than a "just-in-case."

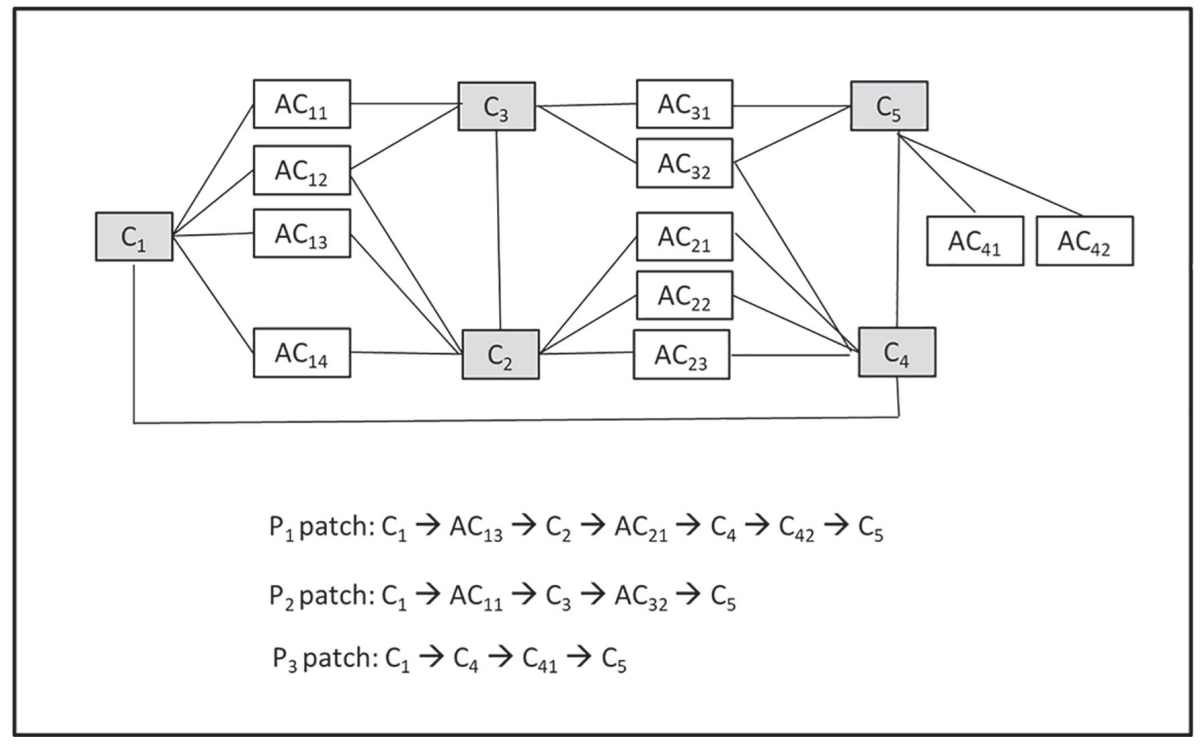

Figure 2. Curriculum patch structure

We envision the IBL course portfolio as supported by faculty, with students being able to consult with faculty members regarding specific PBL problems 
and their relationship to the contents of the IBL curriculum. This should help students decide which modules to select and, should the case arise, encourage the production of new and relevant modules.

\subsubsection{CL}

$\mathrm{CL}$, in the particular form of active small group exercises and discussions remains a key part of our model. The CL periods in the curriculum intervene before and after periods of PBL/IBL learning. They serve to foster collaboration skills, to consolidate student experiences, to reinforce the collective values of the student body, and to instill a sense of self-reflectiveness and orientation among students. Classes might include reflective discussions of the issues and challenges that students have confronted when working with difference and diversity, discussions of the value compromises associated with pursuing a WP like sustainable development, and projective discussions of the future hopes for our society. If most CL learning today involves passive listening, we believe that the CL curricula of the future will maximize the value of face to face encounters and collective discussion. During the CL portion of their education, students will develop a sense of their roles as diverse actors within the unified but diverse movement towards sustainability. They will ask questions and become reflective and responsible engineers.
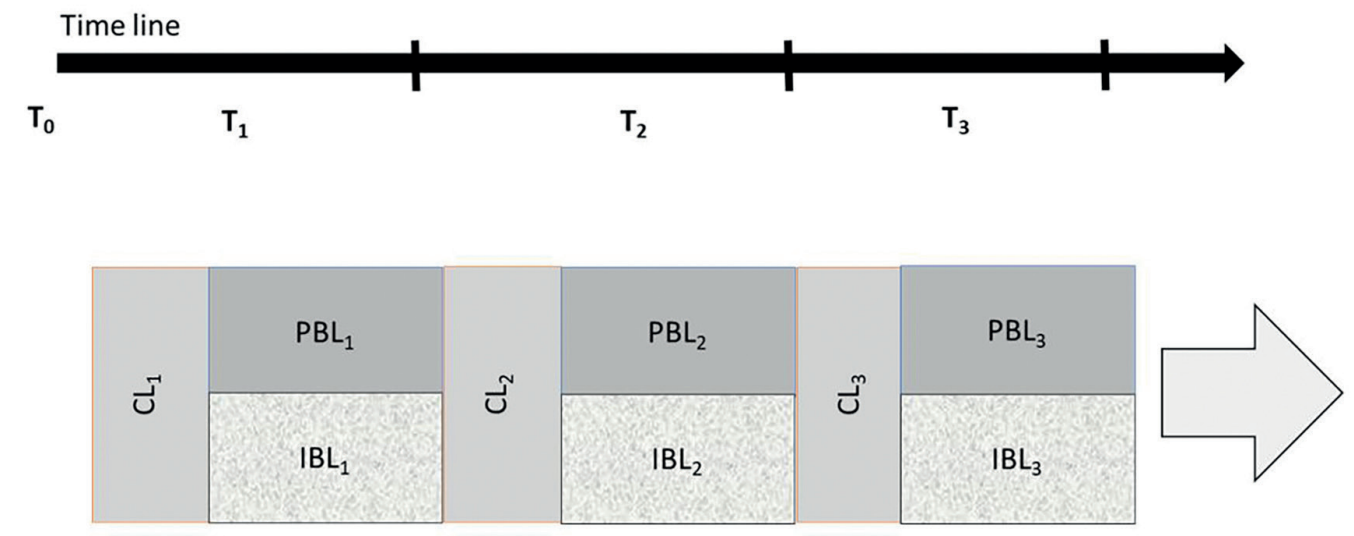

Figure 3. Picturing the curriculum

\subsection{Commentary}

Let us consider how our mixed-medium DCP curriculum meets our challenges:

\subsubsection{Feasibility}

The existence of a similar curriculum at CSU demonstrates feasibility, since what is actual is feasible. The economic advantageousness of our model will increase 
with scale. The start-up costs for IBL are significant: technology investments and pedagogical adaptations are required. Once these initial outlays have been made costs stabilize. Upscaling in the form of sharing course content and generation across Europe-something that is fully possible with this model—and desirable if we are to understand place as a differentiating medium and part of the informal curriculum - will further reduce costs per student. The costs of the PBL pillar will depend on institutional choices relative to whether institution-funded humanitarian and social welfare projects or industry-funded work placements make up the bulk of the PBL curriculum. We feel that the former will be most beneficial for fostering sustainability (if they are also most burdensome on institutional budgets.) The CL aspects of the curriculum should pose no particular challenge given the structure of existing institutions (if large lecture halls may become obsolete). Teaching staff may need to re-skill, and the transition will not necessarily be an easy one. That said, teaching media are better aligned with intended outcomes than in traditional single-medium curricula, which are quite simply artifacts of another age in media technology. Rather that primarily teaching as lecturers (note that this term hearkens back to medieval classrooms in which teachers read out loud due to the unavailability of printed materials), faculty will need to act primarily as expert competence development advisors during the PBL curriculum and facilitators during the CL curriculum. Despite these changes, the relevance of faculty research and expertise will be unchanged. A benefit of using IBL as a medium for lectures is that it provides a possibility for meaningful data collection and analysis relative to teaching effectiveness. Tracking student success as a function of different communicational strategies will permit the data-driven evolution of teacher-centered instructional approaches.

The IBL modules are all assessed for mastery, thus there is no quantitative assessment issue within this model. Moreover, as skills learned via IBL are directly applied to workplace problems, practical mastery of the skills found in transcripts is nearly guaranteed. Moreover, students will have a broad portfolio of projects to demonstrate their competences and to share with potential employers. Nevertheless, student comparison remains challenging for DCP institutions. Different students really are different. However, this may be a good thing. Instead of asking which of two students is "better" in some abstract sense, employers can look at student skill sets when considering employee fit. Should two students of similar profiles find themselves in competition, employers can compare the total number of skills acquired or they can ask former employers and supervisors if they recommend the student. These seem better employee fit predictors than whether student A got a higher average score than student $B$ on a series of standardized but purely academic exercises.

\subsubsection{Optimization of Collaboration}

Our model has a core curriculum. Its three functions include inculcating basic elements of technical literacy, fostering the ability to work together, 
and the inculcation of sustainability-oriented values. Our model curriculum is a learning machine whose curriculum development is driven by the interaction between PBL, IBL module relevance sorting, and CL feedback loops. Different PBL experiences will yield different skill set demands, so the relevance of curricula to future challenges depends upon the specific historical evolution of problems within the school's larger ecosystem. The evolving curricula of a trans-European network of schools could constantly adapt engineering education to the rigors of achieving sustainable development within the European problem ecosystem. Our model specifically takes into account orientation. This is one of core functions of the CL curriculum. Rather than taking their orientation from a top-down administration-generated narrative, during CL classes students orient themselves via reflection on practical experiences, interactions with their peers, and discussions with faculty. This not only encourages ownership over their own education and the collective project of sustainable development but instills in them a sense of responsibility.

Some noteworthy benefits of our curriculum include its solution to the problem of the skills gap; its ability to be scaled up via the sharing of the IBL modules; and its relevance to solving the looming problem of preparing engineers to be life-long learners. Given that many experts predict the rapid evolution of technology over the next decades, it will be important to train engineers in such a way as to prepare them to stay up to date. The feedback loop between encountering practical challenges and preparing to confront them via IBL study is a method that professional engineers can continue employing throughout their entire careers and may well be a key to adapting engineering education to the innovation economy.

\section{Conclusions}

In this paper we have set out to suggest that given the complexity of sustainable development as a problem for future engineers, diversifying curricula are both desirable and feasible. We have presented abstract arguments which draw on complexity theory to demonstrate why this is the case. We have also discussed how mixed media curricula can function to enable the training of competence-diverse engineers. Our model is both preliminary and theoretical, though we hope that it will inspire debate and discussion. With this in mind, let us signal a few questions that seem to merit investigation: Which competences are most pedagogically suitable for which media? Which competences are seminal? What procedures can be used to optimize the curricular contents of each medium? What are the problems generated by the complexification of engineering education set in motion by DCP engineering curricula? 


\section{References}

Brynjolfsson, Erik, and Andrew McAfee. "The Second Machine Age: Work, Progress, and Prosperity in a Time of Brilliant Technologies.” New York: Norton, 2014.

Christensen, Clayton M., Curtis W. Johnson and Michael B. Horn. "Disrupting class: How disruptive innovation will change the way the world learns.” New York: McGraw-Hill, 2010.

Elkington, John, and Iann H. Rowlands. "Cannibals with forks: the triple bottom line of 21st century business.” Alternatives Journal 25, No. 4 (1999): 1-42.

Erhardt, Nicola L., James D. Werbel and Charles B. Shrader. "Board of director diversity and firm financial performance. “ Corporate governance: An international review 11, No. 2 (2003): 102-111.

Fricker, Miranda. "Epistemic injustice: Power and the ethics of knowing." New York: Oxford University Press, 2007.

Graham, Ruth. "The global state of the art in engineering education.” Massachusetts Institute of Technology (MIT) Report, Cambridge MA, USA, 2018.

Krakauer David C. "Robustness in Biological Systems: A Provisional Taxonomy.” In: Deisboeck T.S., Kresh J.Y. (eds) Complex Systems Science in Biomedicine. Topics in Biomedical Engineering International Book Series. New York: Springer, 2006.

Livermore, David. "Driven by Difference: How Great Companies Fuel Innovation Through Diversity.” New York: Amacom, 2016.

McLeod, Poppy Lauretta., Sharon-Alisa Lobel and Taylor H. Cox Jr. "Ethnic diversity and creativity in small groups.” Small group research 27, No. 2 (1996): 248-264.

Minsky, Marvin. "Inventive Minds: Marvin Minsky on Education.” Cambridge MA: MIT Press, 2019.

Mor Barak, Michàlle E. "Inclusion is the key to diversity management, but what is inclusion?." Human Service Organizations: Management, Leadership \& Governance 39, No. 2 (2015): 83-88.

Nelson, Ben, and Stephen S. Kosslyn. "Foundations of the Curriculum. Building the Intentional University: Minerva and the Future of Higher Education.” Cambridge MA: MIT, 2017.

Nest, Michael, “Coltan.” Cambridge, MA: Polity, 2011.

Oliver, Tom H., Matthew S. Heard and Nick JB Isaac et al. "Biodiversity and resilience of ecosystem functions.” Trends in Ecology \& Evolution 30, No. 11 (2015): 673-684.

Page, Scott E. “The Difference.” Princeton: Princeton University Press, 2007.

Page, Scott E. ”Diversity and complexity (Vol. 2).” Princeton, NJ: Princeton University Press, 2010. Page, Scott E. “The Diversity Bonus.” Princeton, NJ: Princeton University Press, 2018.

Quelhas, Osvaldo Luiz Gonçalves, Gilson Brito Alves Lima, Nicholas Van-Erven Ludolf, et al. "Engineering education and the development of competencies for sustainability." International Journal of Sustainability in Higher Education 20, No. 4 (2019): 614-629.

Richard, Orlando C. "Racial diversity, business strategy, and firm performance: A resource-based view.” Academy of Management Journal 43, No. 2 (2000): 164-177.

Rittel, Horst W. J., and Melvin M. Webber. "Dilemmas in a general theory of planning.” Policy Sciences 4, No. 2 (1973): 155-169.

Robinson, Gail, and Kathleen Dechant. "Building a business case for diversity.” Academy of Management Perspectives 11 (1997), Vol. 3: 21-31. 
Runco, Mark A., and Garrett J. Jaeger. "The standard definition of creativity." Creativity Research Journal 24, No. 1 (2012): 92-96.

Sawyer, Keith, “Group Genius: The Creative Power of Collaboration.” New York: Basic Books, 2007.

Sunstein, Cass R., and Reid Hastie. "Wiser: Getting beyond groupthink to make groups smarter.” Cambridge, MA: Harvard Business Press, 2015.

Surowiecki, James. "The wisdom of crowds.” New York: Anchor, 2005.

Tabas, Brad, Una Beagon and Klara Kövesi. "Report on the future role of engineers in the society and the skills and competences required for engineers.” First Project Report, A-STEP 2030 project, 2019: 1-40.

Whitacre, James M., and Axel Bender. "Networked buffering: a basic mechanism for distributed robustness in complex adaptive systems." Theoretical Biology and Medical Modelling 7, No. 1 (2010): 1-20. https://doi.org/10.1186/1742-4682-7-20 